\title{
Deformation of the contact line around spherical particles bound at anisotropic fluid interfaces $\dagger$
}

\begin{abstract}
Nesrin Şenbil ${ }^{\mathrm{ab}}$ and Anthony D. Dinsmore (D)*a
When a particle adsorbs at a liquid interface, the 3-phase contact line geometry depends on the shape of the particle and of the liquid interface. The shape of the contact line is the key to controlling capillary forces among particles, and is therefore a useful means to direct assembly of interfacial particles. We measured the shape of the contact line around millimeter-sized PDMS-coated glass spheres at water/air interfaces with anisotropic shapes. We studied the advancing and receding conditions separately. We focused on interfaces with a cylindrical shape, where the predominant deformation of the meniscus and the contact line both have quadrupolar $\cos (2 \phi)$ symmetry. We related the measured magnitude of the quadrupolar deformation to the applied vertical force on the sphere and the interface's deviatoric curvature, $D_{0}$. For modest curvature $\left(D_{0}<0.1 \times\right.$ sphere radius), our results agree with the theoretical prediction for free particles. At higher curvature, the measurements exceed the theory. The theory appears to apply even when there is contact-angle hysteresis, as long as the measured contact angle is used rather than the equilibrium (Young-Dupré) angle. The magnitude of the quadrupolar deformation depends on the applied force. Together, these results show the range of validity of the theory.
\end{abstract}

\section{Introduction}

The binding of particles at fluid interfaces is an active area of research because of its frequent appearance in nature and technology. Technologically important examples abound in the form of particle-coated droplets, known as Pickering emulsions, which are found in such applications as foods, foams, particlebased micro-capsules, and dispersants for oil-spill cleanup. ${ }^{1-9}$ Particles bind at the fluid interface to minimize the interfacial energy. ${ }^{10}$ This binding may be accompanied by a deformation of the fluid interface depending on particle size, roughness, ${ }^{11}$ shape, ${ }^{12-14}$ and weight, ${ }^{15}$ or interface shape. This deformation, in turn, generally leads to lateral interactions with other particles, mediated by the interface. ${ }^{11,15,16}$ In addition to aiding the rapid aggregation of particles and controlling the mechanical properties of particle-laden interfaces, ${ }^{17}$ these capillary forces can also guide the assembly of floating components when fabricating macroelectronic devices. ${ }^{18-21}$ In all of these cases, the key to controlling the capillary interaction is the shape of the contact line where the fluid interface meets the particle's surface.

Predictions of the shape of the fluid interface generally start with the assumption that the three-phase contact angle $\theta$ is

\footnotetext{
${ }^{a}$ Department of Physics, Univ. of Massachusetts, Amherst, MA, 01003, USA. E-mail: dinsmore@physics.umass.edu

${ }^{b}$ Department of Physics, Univ. of Fribourg, Fribourg 1700, Switzerland

$\dagger$ Electronic supplementary information (ESI) available. See DOI: 10.1039/ c7sm01548h
}

uniform and equal to the equilibrium (Young-Dupré) value. The shape may then be found by requiring that the free fluid interface have constant mean curvature (ignoring the effect of gravity on the fluids). Defining $u(x, y)$ as the interface height at every point above a reference plane and assuming a weak deformation $(|\nabla u| \ll 1)$, the mean curvature of the interface is $\frac{1}{2} \nabla^{2} u .^{15,16}$ The equation defining the interface shape resembles Poisson's equation of electrostatics in two dimensions for a uniform charge density. As in electrostatics, the height field can be written as a superposition of monopole (isotropic $u \sim \ln (r)$ ), dipole $\left(u \sim r^{-1} \cos \phi\right)$, quadrupole $\left(u \sim r^{-2} \cos (2 \phi)\right)$ and higher terms. ${ }^{16,22,23}$ When a spherical particle binds at a planar interface, no deformation appears unless an applied force acts on the particle (e.g. from gravity). ${ }^{15,24,25}$ For particles with an anisotropic shape, however, the constant-contact angle condition leads to deformations even without any applied force. These deformations depend on the particle shape and have been studied for rods, ${ }^{13}$ ellipsoids ${ }^{12,26,27}$ and cuboids, ${ }^{28,29}$ and spheres with non-uniform pinning surfaces. ${ }^{11,30}$

When the fluid interface itself is curved with an anisotropic shape, force-free spherical particles will also deform the interface. The theory was first developed by Würger ${ }^{22}$ for zero-meancurvature interfaces (catenoids). Zeng et al. ${ }^{31}$ then considered other interface shapes and predicted a general form of the force between two free spheres, which is induced by the interface shape. For a sphere at a cylindrical interface, these calculations predict a quadrupolar deformation, in which the contact line height is $z(\phi)=r_{\mathrm{c}}^{2} \cos (2 \phi) /(12 R)\left(1+O\left(r_{\mathrm{c}} / R\right)^{2}\right)$, where $R$ is the 

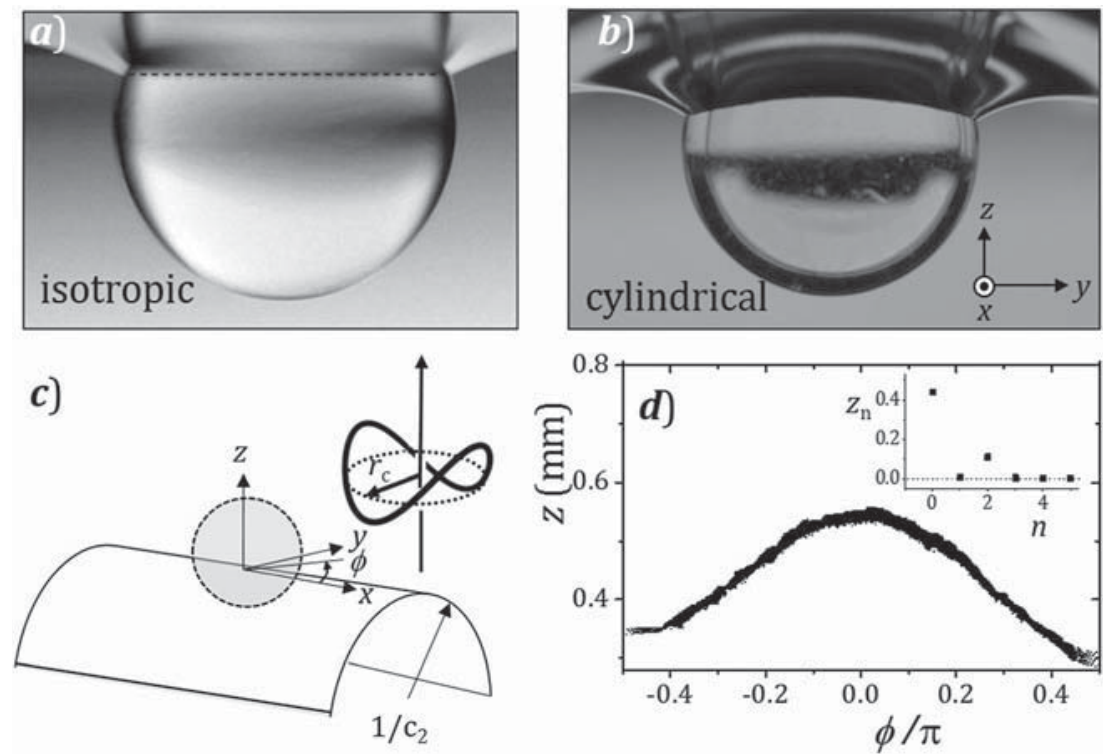

Fig. 1 (a) A glass sphere at an isotropic air-water interface, where the contact line is a ring. (b) A glass sphere at a cylindrical air-water interface $\left(D_{0}=\right.$ $0.21 \mathrm{~mm}^{-1}$ ), where the contact line is deformed. The image is looking along the cylinder's axis. (c) Schematic of the cylindrical interface showing the coordinates and the contact radius, $r_{\mathrm{c}}$. (d) The height of the contact line, $z(\phi)$, measured around the sphere shown in (b). Inset of d: $z_{n} v s$. $n$.

cylinder radius, $r_{\mathrm{c}}$ is the radius of the contact line at mean height, and $\phi$ is the polar angle measured in the plane of the interface (Fig. 1c). ${ }^{22,31}$ The contact line resembles the rim of a saddle or of a Pringles ${ }^{\circledR}$ potato chip.

For capillary interactions, previous authors have noted that the essential feature of the interface shape is the anisotropy of the curvature, which is appropriately parameterized by the deviatoric curvature, ${ }^{32,33} D \equiv \frac{1}{2}\left(c_{1}-c_{2}\right)$, where $c_{1,2}$ are the two principal curvatures and the $x$-axis is chosen to lie parallel to the principal-curvature direction defined by $c_{1}$. Curvatures are positive when the center of the arc lies at positive $z$. Rewriting the earlier result, the predicted shape of the contact line around a spherical particle is then $z(\phi)=(1 / 6) D_{0} r_{\mathrm{c}}^{2} \cos (2 \phi)$, where $D_{0}$ is the deviatoric curvature prior to insertion of the spherical particle. In the language of electrostatics, the quadrupolar deformation is induced by the effective field $D_{0}$. The linear dependence on $D_{0}$ captures the magnitude and the symmetry. For example, rotating the interface by an angle of $\pi / 2$ causes the deformation to change sign (upward regions of the quadrupolar deformation become downward) and also corresponds to swapping the labels $c_{1}$ and $c_{2}$, and hence changing the sign of $D_{0}$. This symmetry property also accounts for the observation that a rod-shaped particle (which creates a quadrupolar deformation that is fixed with respect to the particle's frame of reference) rotates by $\pi / 2$ when the curvature of the interface is inverted. ${ }^{13}$

Experimental evidence for the quadrupolar deformation came from the observation that colloidal spheres adopted square-symmetry lattices when bound at a fluid interface with $D_{0} \neq 0$ (saddles, cylinders), which matches the predicted pattern of inter-particle capillary forces. ${ }^{33}$ While these results establish the qualitative point regarding the symmetry of the deformation, there are still no direct measurements of the deformation itself. Hence, we need to test the most basic assumptions that the contact angle is uniform and equal to the Young-Dupré value. Moreover, we need to investigate the role of an applied force normal to the interface and explore the range where the interface slope is not small. Furthermore, we need to test whether hysteresis in the contact angle - so common in real materials - affects the deformation shape.

In this article, we report measurements of the shape of a contact line around a millimeter-scale spherical particle that was held at various positions astride an air-water interface. We measured the responses for both advancing and receding contact lines, for which the contact angles differed. We used PDMS-coated glass spheres and created air-water interfaces that were planar $\left(D_{0}=0\right)$ or cylindrical $\left(D_{0} \neq 0\right)$ in shape. The shape of the air-water-particle contact line, $z(\phi)$, was measured and fit to a multipole expansion. The only non-negligible terms corresponded to the monopole (isotropic) and quadrupolar symmetries. First, we found that the quadrupolar term, $z_{2}$, depends on the vertical component of the force applied to the particle: $z_{2}$ reached a peak value when the contact line was near the equator of the sphere. Thus, the vertical force can affect deformation modes other than the isotropic $(n=0)$ one. Second, after extracting the $z_{2}$-values for zero applied force (which we call $z_{2}^{0}$ ), we found that these values agree reasonably well with the zero-force prediction: $z_{2}^{0}=(1 / 6) D_{0} r_{\mathrm{c}}{ }^{2}$. The data are indistinguishable from theory when $D_{0} a$ is less than approximately 0.1 , but exceed the theory at larger $D_{0} a$. Third, we found that the measured contact angles $(\theta), r_{\mathrm{c}}$, and $z_{2}$ are different for advancing and receding contact lines. However, in both cases the $z_{2}$ varies with $D_{0} r_{\mathrm{c}}{ }^{2}$ in the same way. Thus, it appears that the contact angle only sets the contact radius $r_{\mathrm{c}}$, and it does not matter whether or not the angle is the equilibrium value. The deviation from the theory as $D_{0} a$ approaches 1 and the dependence on the vertical applied force remain topics for further investigation. 


\section{Materials and methods}

\section{Preparation of PDMS-coated spheres}

We used borosilicate glass spheres with a diameter of $1 / 8$ inch (radius $a=1.59 \mathrm{~mm}$ ), manufactured by Winsted Precision Ball Company and purchased from McMaster-Carr (cat. no. 8996K22). Spheres were first soaked in a NoChromix ${ }^{\circledR} /$ sulfuric acid mixture overnight and washed with de-ionized (DI) water many times, then left in DI water for another $2 \mathrm{~h}$, then dried in an oven at $80{ }^{\circ} \mathrm{C}$. A rigid rod was attached to the sphere using epoxy and allowed to cure for at least $2 \mathrm{~h}$. To coat the sphere with PDMS, we followed the procedure described previously ${ }^{17,34,35}$ and summarized here. The rod-attached sphere was dipped into a vial $(20 \mathrm{~mL}$ borosilicate glass scintillation vials, Fisher Scientific, cat. no. 03-337-4) filled with PDMS (trimethylsiloxy terminated PDMS, $94 \mathrm{kDa}$; Gelest cat. no. DMS-T22) and heated in an oven for $24 \mathrm{~h}$ at $150{ }^{\circ} \mathrm{C}$. The rod-attached sphere was then removed from the oven, allowed to cool to room temperature, and washed with toluene, acetone and DI water in that order. With care, in most cases this preparation process led to smooth contact lines with no defects or discontinuities visible with $\sim 5 \mu \mathrm{m}$ image resolution. An atomic-force microscopy image of a treated sphere was reported previously. ${ }^{35}$ In some cases, however, the spherical particle showed a defect that disrupted the contact line; in these cases, the sphere was discarded. Three different glass spheres of the same size were used in these experiments and no differences among the results were found.

\section{Preparation of cylindrical interfaces}

Two razor blades were used to pin the interface and create a curved shape. The sharp edges of the two razor blades (of length $L \sim 5 \mathrm{~cm}$ ) were placed facing one another, separated by $7.5 \mathrm{~mm}$. When the container was filled with deionized water, the interface was pinned at the sharp edges. As more water was added, the interface adopted the shape of a hemicylinder, as shown in the schematic of Fig. 1c. The razor blades held one principal curvature $c_{1}=0$. We found the other principal curvature $c_{2}$ by taking an image of the clean interface before the sphere was placed, and we fit the interface line to a circle of radius $-1 / c_{2}$ (where $c_{2}$ is negative because the center of the arc lies below the plane $z=0$ ). Because $L \gg a$, the interface shape was uniform across a region much larger than the spherical particle.

\section{Procedure}

The rod-attached sphere was attached to a translation stage mounted above the water. The sphere was displaced into the fluid interface by small amounts $(\sim 0.1 \mathrm{~mm})$. Between displacements, we waited about $5 \mathrm{~s}$ for the water flow to cease and the contact line to reach a steady state before acquiring an image. As we reported earlier, the measured contact angle and contact-line shape did not change between $1 \mathrm{~s}$ and approx. 5 min. ${ }^{35}$ After the sphere was nearly fully immersed in water, it was pulled back following exactly the same procedure until it detached from the interface. Pushing the sphere into the interface or pulling it out of the interface corresponds to advancing and receding of the interface across the non-wet surface of the sphere, respectively. These two cases are discussed separately because they exhibit different contact angles and deformations.

\section{Measuring the shape of the interface and contact line}

The glass sphere and the air-water interface were imaged using a Nikon D5100 digital camera with a $60 \mathrm{~mm}$ lens and $68 \mathrm{~mm}$ extension tubes. The camera was located along the $x$ - or $y$-axis. The contact line was imaged through the air when the meniscus curved upward, and through the water when it curved downward (as in Fig. 1a and b). The images show contrast between the sphere and the interface. The $(x, z)$ or $(y, z)$ coordinates of the interface and of the contact line were found using the 'Find Edges' algorithm in Image,${ }^{36}$ followed by a thresholding process. Since we know that the contact line lies on the surface of the sphere, we converted the coordinates to $z$ vs. $\phi$ (the polar angle in the $x-y$ plane) as defined in Fig. $1 c$ and d.

\section{Results and discussion}

The image in Fig. 1a shows that an initially planar interface yields a contact line that is always planar and circular, irrespective of the immersion depth of the sphere. In contrast, an initially cylindrical interface yields a smooth but non-circular contact line (Fig. 1b).

Following the electrostatic analogy, we plotted the measured height of the contact line as a function of the polar angle, $z(\phi)$, and fit the result to the following function:

$z(\phi)=z_{0}+z_{1} \sin (\phi)+z_{2} \cos (2 \phi)+z_{3} \sin (3 \phi)+z_{4} \cos (4 \phi)+z_{5} \sin (5 \phi)$.

We chose the sine and cosine terms that preserve the known reflection symmetry of the interface, $x \rightarrow-x$. The first term, $z_{0}$, is the average height of the contact line relative to the sphere's center. The contact radius, $r_{\mathrm{c}}$, is the radius of the sphere measured at the height $z_{0}$ (Fig. 1c). The terms $n=1,2$ correspond to the dipole and quadrupole, respectively. For the initially planar interface, we found that $z(\phi)$ is a constant and $z_{n}$ is indistinguishable from zero for all $n>0$.

For the cylindrical interface with $D_{0}=0.21 \mathrm{~mm}^{-1}$, Fig. $1 \mathrm{~b}$ shows the image and Fig. 1d shows $z(\phi)$ and the values of $z_{n}$ resulting from the fit. (An additional example, tabulated data, and a discussion of a small tilt offset are given in the ESI $\dagger$.) We found that only the isotropic and quadrupolar $(n=0,2)$ terms differed substantially from zero. This finding is consistent with the reflection symmetry about the $x z$ and $y z$ planes (which eliminates the odd- $n$ terms) and the intuitive expectation that the deformations at $\phi=0$ and $\pi / 2$ should differ owing to the different principal curvatures (which contradicts the $n=4$ term).

At cylindrical interfaces, the magnitude of the quadrupolar $z_{2}$ term changes with $D_{0}$, and also with the depth of immersion in the interface. After the sphere was immersed deeper in the water, the contact line shifted toward the upper pole while maintaining a constant contact angle. (Example images are shown in Fig. S1 of the ESI $\dagger$.) For each interface shape studied, we found that $z_{2} / a$ reached a maximum when the contact lay 


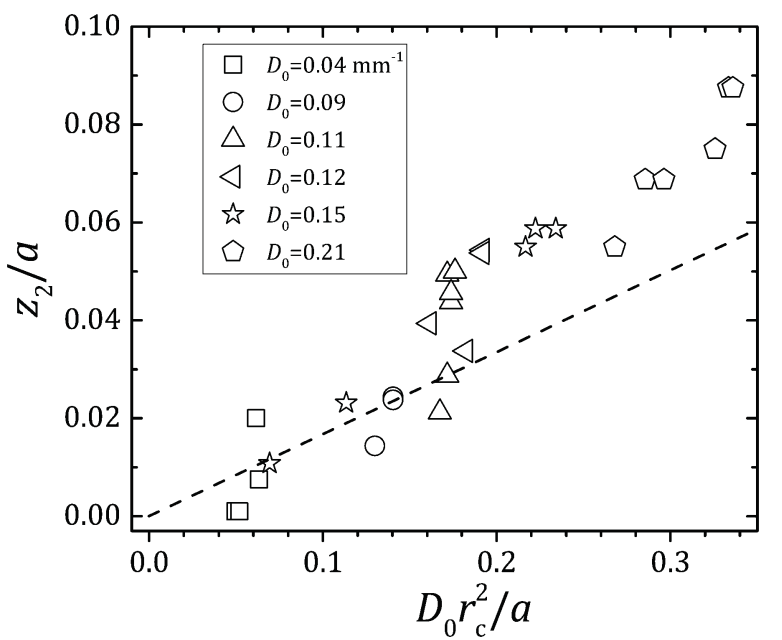

Fig. 2 Measured $z_{2} / a$ for different spheres, immersed at various depths, and at different $D_{0}$ ranging from 0.04 to $0.21 \mathrm{~mm}^{-1} . z_{2} / a$ is plotted against $r_{\mathrm{c}}^{2} D_{0} / a$, which accounts for the interface shape. The dashed line shows the theory of Zeng et al. for the case of zero capillary force, which does not adequately explain the data: the applied force and immersion depth affect $z_{2}$ in a way that is not accounted for by this $r_{c}$ scaling. For clarity, only the receding data are shown but advancing data follow the same trend.

near the equator $\left(r_{\mathrm{c}} / a=1\right)$. We also found that the $z_{2} / a$ values differed for receding and advancing cases. (Data are shown in Fig. S3 of the ESI + .) We return to this point below.

To highlight the effect of interface shape, Fig. 2 shows a plot of $z_{2} / a$ as a function of the initial deviatoric curvature and the contact radius, rescaled as $r_{\mathrm{c}}^{2} D_{0} / a$. This form is the predicted scaling when there is zero applied force. As shown in Fig. 2, the theory roughly captures the overall trend of the data. However, the data show that for a fixed value of $D_{0}$ there is a strong trend of increasing $z_{2}$ with $r_{\mathrm{c}} / a$. We attribute the scatter in the data and the deviation from the theory to a net capillary force acting on the sphere in the vertical direction. The figure also shows that the force has a substantial effect on the $z_{2}$ value that is not accounted for by the contact radius $r_{\mathrm{c}}$.

As illustrated in Fig. 3, the rod applied a force that was equal and opposite to the force applied by the interface. The latter, which we call $F_{\text {cap }}$, arises from the air-water interface pulling along its tangent. ${ }^{16,37}$ We calculated $F_{\text {cap }}$ from the air-water interfacial tension $\left(\gamma=72 \mathrm{mN} \mathrm{m}^{-1}\right)$ and the known shape of the contact ring. The force is given by $\gamma \oint \mathrm{d} s \hat{\boldsymbol{t}}(s) \cdot \hat{\boldsymbol{k}}$, where the integral is done around the contact line, $\mathrm{d} s$ is the differential length, and $\hat{\boldsymbol{t}}(s)$ is the unit vector that lies in the plane of the air-water interface and perpendicular to the contact line at position $s$. The direction of $\hat{\boldsymbol{t}}$ around the contact ring was obtained numerically from the measured contact-line shape by requiring that the interface have a uniform mean curvature and uniform contact angle. We then integrated to find $F_{\text {cap }}$ for each position of the sphere.

Fig. 3 shows the measured quadrupole deformation as a function of the calculated $F_{\text {cap }}$ for one interface. To facilitate comparison to the theory, we normalized $z_{2}$ by $r_{\mathrm{c}}^{2} D_{0}$ and normalized $F_{\text {cap }}$ by the characteristic force scale $2 \pi \gamma a$ (which is also the maximum possible value of $\left.F_{\text {cap }}\right)$. The data show that

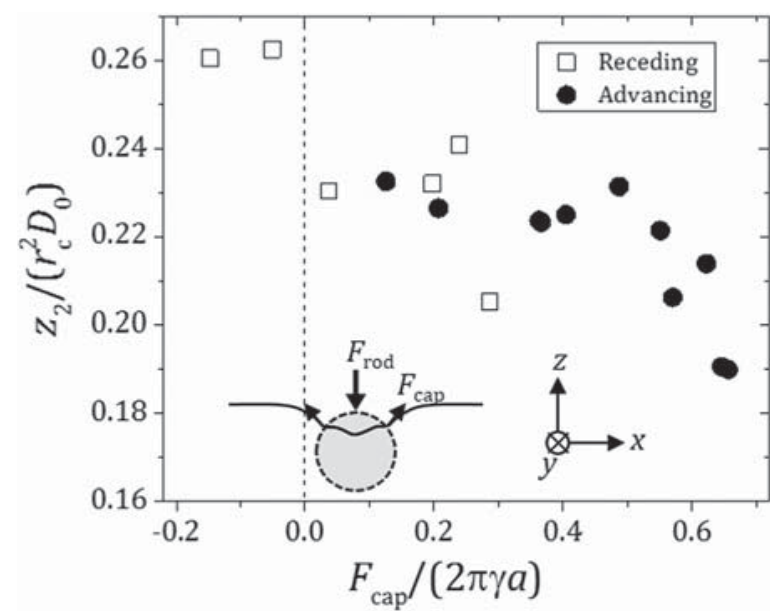

Fig. 3 Measured quadrupole deformations as a function of the calculated capillary force $F_{\text {cap. }}$. Data are plotted in the normalized, dimensionless form. These measurements are for $D_{0}=0.21 \mathrm{~mm}^{-1}$ at various immersion depths. $F_{\text {cap }}$ was obtained numerically from the measured interface shape. The inset illustrates the force applied by the rod attached to the sphere, which balances the capillary force $F_{\text {cap }}$

a larger $F_{\text {cap }}$ leads to a smaller $z_{2} / r_{\mathrm{c}}^{2} D_{0}$. The same result was found in all cases that we studied.

Why would an applied force change the magnitude of the quadrupolar deformation? In terms of the electrostatic analogy, this question becomes: why would the $n=0$ and $n=2$ modes couple to one another? In the limit of small interface slopes and small particles $\left(a D_{0} \ll 1\right)$, the constant-contact angle boundary condition on the cylindrical surface constrains the $n=2$ mode but not the isotropic $(n=0)$ one. ${ }^{31}$ Hence, there should be no coupling between $n=0$ and $n=2$ modes. In our experiments, however, $a D_{0}$ was not always small (it reached 0.33 ), so that the correct boundary condition may include terms that involve both $z_{0}$ and $z_{2}$. Moreover, the interface slope was not always small, so that the linear approximation described in the introduction may not always be accurate. The slope is evident from the images of Fig. 1 and Fig. S2 (ESI $\dagger$ ), and also from the $F_{\text {cap }} / 2 \pi \gamma a$ values. For a planar interface, $F_{\text {cap }} / 2 \pi \gamma a$ is the average sine of the tilt angle of the interface at the contact line. In many of our data points, $F_{\text {cap }} / 2 \pi \gamma a$ exceeds 0.6 . Predicting the shape of the interface in this strong-deformation regime is beyond the scope of this article but would be an interesting topic of future study.

To compare the measured deformations to the zero-force theory, we extrapolated the data to $F_{\text {cap }}=0$ and found the corresponding deformation (which we call $z_{2}^{0}$ ). We used two different methods to find $z_{2}^{0}$. In the first method, we calculated $F_{\text {cap }}$ from images using the numerical method described above, and then extracted $z_{2}^{0}$ from a plot of $z_{2} v s$. $F_{\text {cap }}$. In the second method, we estimated where the capillary force is zero by calculating the contact radius $\left(r_{\mathrm{c}}^{0}\right)$ at which the deformation would be zero at an initially planar interface. This yields the condition $r_{\mathrm{c}}^{0}=a \sin \left(2 \pi-\theta_{\mathrm{A}, \mathrm{R}}\right)$, where $\theta_{\mathrm{A}, \mathrm{R}}$ is the measured contact angle, which is different for the advancing (A) and receding (R) cases. Here we used the $\theta_{\mathrm{A}}$ and $\theta_{\mathrm{R}}$ values measured 
for each experiment. We found $\theta_{\mathrm{A}}=110^{\circ}$, independent of interface shape and immersion depth. ${ }^{35}$ As we reported in an earlier study with similar spheres, $\theta_{\mathrm{R}}=101^{\circ}$ at planar interfaces but, surprisingly, decreases as $D_{0}$ increases, reaching $80^{\circ}$ when $D_{0}=0.21 \mathrm{~mm}^{-1} \cdot{ }^{35} \mathrm{An}$ example of each method is shown in Fig. S4 of the ESI. $\dagger$

Having removed the effect of the applied force, we plotted the extracted $z_{2}^{0} / a$ as a function of $D_{0} r_{\mathrm{c}}{ }^{2} / a$, as suggested by the theory. The results are shown in Fig. 4 for various anisotropic interfaces with $D_{0}$ ranging from 0 to $0.21 \mathrm{~mm}^{-1}$. Our two methods of finding $z_{2}^{0}$ are consistent with one another. We find a good agreement with the theory when $D_{0} r_{\mathrm{c}}{ }^{2} / a$ and $z_{2} / a$ are small. However, the measured deformations exceed the theory when $D_{0} r_{\mathrm{c}}{ }^{2} a$ exceeds approximately 0.15 (or $D_{0}$ exceeds approximately $0.1 / a$ ). This disagreement is not surprising because the theory of Zeng et al. relied on the assumption that $D_{0} a \ll 1,{ }^{31}$ so that nonlinear effects associated with finite $D_{0} a$ might be important in our experiment. It might also be that the force of gravity acting on the air-water interface (which was neglected by the theory) affects the quadrupole magnitude. In our experiments, the capillary length, beyond which scales gravity predominates, is given by $\left(\gamma /\left(g \rho_{\mathrm{w}}\right)\right)^{1 / 2}=2.7 \mathrm{~mm}$, where $\rho_{\mathrm{w}}$ is the mass density of water and $g$ is the acceleration due to gravity. ${ }^{37}$ Finally, the zero-displacement boundary condition along the razor blades is not the same as the boundary-free geometry considered in the theory and might affect $z_{2}$. In any case, we are unable to explain why the measured values were systematically greater than the theory in this strong-deformation regime. Fig. 4 shows no systematic difference between the results for advancing and receding contacts, even though the corresponding contact angles differed by as much as $30^{\circ}$. The theory explicitly assumed that the contact angle has the equilibrium value (which must lie between $\theta_{\mathrm{R}}$ and $\theta_{\mathrm{A}}$ ). The theory also predicts that the only role of $\theta$ is to set the equilibrium contact radius $\left(r_{\mathrm{c}}=a \sin \theta\right.$ if $\left.D_{0} a \ll 1\right)$. From our data, it appears that

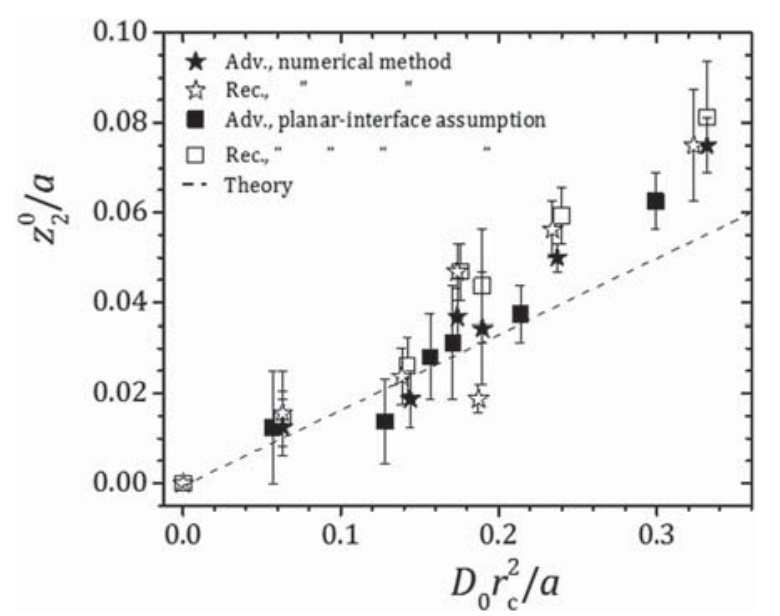

Fig. 4 Plot of the zero-capillary force quadrupole amplitude, $z_{2}^{0} / a$, as a function of $D_{0} r_{c}^{2} / a$. The symbols stars and squares correspond to the numerical calculation of $F_{\text {cap }}$ and the planar-interface assumption, respectively. Filled and open symbols denote the advancing (adv.) and receding (rec.) cases, respectively. The dashed line shows the theory of Zeng et al. for the case of zero capillary force. this result also applies to the case of uniform but non-equilibrium contact angles.

In some cases, we measured the contact angles at the four symmetry positions $\left(\phi=0^{\circ}, 90^{\circ}, 180^{\circ}\right.$ and $\left.270^{\circ}\right)$. In such cases, these angles were indistinguishable from one another. As we noted previously, however, ${ }^{35}$ our images cannot accurately provide the contact angles at arbitrary points on the contact line: the contact line is not always normal to the image plane, so that the two rays that define $\theta$ do not always lie in the image plane. Nonetheless, because $\theta$ is consistent at the four symmetry positions, it seems most likely that $\theta$ is uniform around the ring.

\section{Summary and conclusions}

In summary, we measured the contact line shape around smooth, non-pinning spheres that were immersed partially into a planar or cylindrical interface. The magnitude of the curvature-induced deformation is important because it affects the energy of binding, it induces lateral capillary forces between two spheres, ${ }^{31,33}$ and it induces a lateral force on a sphere at an interface with a non-uniform shape. ${ }^{30,31,38}$ We found that the deformation around a sphere at an initially planar interface is isotropic (as expected), but the deformation at an initially cylindrical interface is predominantly quadrupolar. The magnitude

$\left(z_{2}\right)$ depends on $r_{\mathrm{c}}, D_{0}$ and $F_{\text {cap. }}$

When $F_{\text {cap }} \rightarrow 0$ and $D_{0} a<0.1$, we found good agreement with the equilibrium theory of Zeng et al. for the zero-force deformation $z_{2}^{0} \cdot{ }^{31}$ When $D_{0} a$ was greater than approximately 0.1 , the $z_{2}^{0}$ exceeded the theoretical prediction. The difference was about $30 \%$ when $D_{0} a=0.33$. The measured $z_{2}$ also exceeded the theory when the sphere was pulled outward from the center of the cylinder by an external force (i.e., $F_{\text {cap }}<0$ ). In both these cases, the slope was not small, so that the superposition approximation and the electrostatic analogy break down.

Our spherical particles had $\theta_{\mathrm{A}}-\theta_{\mathrm{R}}$ as large as $30^{\circ}$ but with smooth contact lines and no discernible pinning of the contact line. In the regime $D_{0} a<0.1$, the equilibrium theory successfully predicted the deformation, $z_{2}^{0} /\left(D_{0} r_{\mathrm{c}}^{2}\right)$, despite the contactangle hysteresis. This experimental result significantly expands the utility of the equilibrium theory, since contact-angle hysteresis is almost always present with solid particles and surfaces. ${ }^{37}$

In future work, it would be interesting to study the case of strong curvature $\left(D_{0} a>0.1\right)$, where the linear electrostatic analogy breaks down. To account for practical situations, it would also be useful to study particles with rough surfaces to see whether the predominant deformation at long range still agrees with the smooth-particle, equilibrium theory. In the rough-particle case, the deformation may become time dependent owing to aging of the contact line. ${ }^{39}$

\section{Conflicts of interest}

There are no conflicts to declare. 


\section{Acknowledgements}

This research was supported in part by a grant from $\mathrm{BP} / \mathrm{The}$ Gulf of Mexico Research Initiative through the C-MEDS consortium, and in part by the National Science Foundation (CBET0967620 and CBET-1438425). We thank Vincent Demery for providing us with the python code that computes capillary forces and we thank Wei He for helpful discussions.

\section{References}

1 S. U. Pickering, J. Chem. Soc., 1907, 91, 2001-2021.

2 B. P. Binks, Curr. Opin. Colloid Interface Sci., 2002, 7, 21-41.

3 R. Aveyard, B. P. Binks and J. H. Clint, Adv. Colloid Interface Sci., 2003, 100, 503-546.

4 B. P. Binks and R. Murakami, Nat. Mater., 2006, 5, 865.

5 V. N. Paunov, O. J. Cayre, P. F. Noble, S. D. Stoyanov, K. P. Velikov and M. Golding, J. Colloid Interface Sci., 2007, 312, 381-389.

6 H. P. Zhang, M. Khatibi, Y. Zheng, K. Lee, Z. K. Li and J. V. Mullin, Mar. Pollut. Bull., 2010, 60, 1433.

7 A. Saha, A. Nikova, P. Venkataraman, V. T. John and A. Bose, ACS Appl. Mater. Interfaces, 2013, 5, 3094-3100.

8 V. Garbin, Phys. Today, 2013, 66, 68-69.

9 J. Sun and X. L. Zheng, J. Environ. Monit., 2009, 11, 1801-1809.

10 P. Pieranski, Phys. Rev. Lett., 1980, 45, 569-572.

11 D. Stamou, C. Duschl and D. Johannsmann, Phys. Rev. E: Stat. Phys., Plasmas, Fluids, Relat. Interdiscip. Top., 2000, 62, 5263.

12 J. C. Loudet, A. G. Yodh and B. Pouligny, Phys. Rev. Lett., 2006, 97, 018304.

13 E. P. Lewandowski, J. A. Bernate, P. C. Searson and K. J. Stebe, Langmuir, 2008, 24, 9302.

14 L. Botto, L. Yao, R. L. Leheny and K. J. Stebe, Soft Matter, 2011, 8, 4971.

15 D. Vella and L. Mahadevan, Am. J. Phys., 2005, 73, 817.

16 P. A. Kralchevsky and K. Nagayama, Adv. Colloid Interface Sci., 2000, 85, 145-192.

17 W. He, N. Senbil and A. D. Dinsmore, Soft Matter, 2015, 11, 5087-5094.

18 R. J. Knuesel and H. O. Jacobs, Proc. Natl. Acad. Sci. U. S. A., 2010, 107, 993-998.

19 S. Biswas, M. Mozafari, T. Stauden and H. O. Jacobs, Micromachines, 2016, 7, 54.
20 J. M. Carballo, Q. Ni, J. Vasquez and N. B. Crane, Colloids Surf., A, 2017, 512, 118-128.

21 M. Mastrangeli, Q. Zhou, V. Sariola and P. Lambert, Soft Matter, 2017, 13, 304-327.

22 A. Wurger, Phys. Rev. E: Stat. Phys., Plasmas, Fluids, Relat. Interdiscip. Top., 2006, 74, 041402.

23 J. Leandri and A. Wurger, J. Colloid Interface Sci., 2013, 405, 249-255.

24 L. Zhang, L. Ren and S. Hartland, J. Colloid Interface Sci., 1996, 180, 493-503.

25 M. M. Nicolson, Proc. Cambridge Philos. Soc., 1949, 45, 288-295.

26 S. Coertjens, P. Moldenaers, J. Vermant and L. Isa, Langmuir, 2014, 30, 4289-4300.

27 E. A. van Nierop, M. A. Stijnman and S. Hilgenfeldt, Europhys. Lett., 2005, 72, 671.

28 G. Soligno, M. Dijkstra and R. van Roij, Phys. Rev. Lett., 2016, 116, 258001.

29 T. G. Anjali and M. G. Basavaraj, Langmuir, 2017, 33, 791-801.

30 N. Sharifi-Mood, I. B. Liu and K. J. Stebe, Soft Matter, 2015, 11, 6768-6779.

31 C. Zeng, F. Brau, B. Davidovitch and A. D. Dinsmore, Soft Matter, 2012, 8, 8582-8594.

32 M. Cavallaro, L. Botto, E. P. Lewandowski, M. Wang and K. J. Stebe, Proc. Natl. Acad. Sci. U. S. A., 2011, 108, 20923-20928.

33 D. Ershov, J. Sprakel, J. Appel, M. A. C. Stuart and J. van der Gucht, Proc. Natl. Acad. Sci. U. S. A., 2013, 110, 9220-9224.

34 J. W. Krumpfer and T. J. McCarthy, Faraday Discuss., 2010, 146, 103.

35 N. Şenbil, W. He, V. Démery and A. D. Dinsmore, Soft Matter, 2015, 11, 4999-5003.

36 W. S. Rasband, ImageJ, U. S. National Institutes of Health, Bethesda, Maryland, USA, 1997-2016, https://imagej.nih. gov/ij/.

37 D. Bonn, J. Eggers, J. Indekeu, J. Meunier and E. Rolley, Rev. Mod. Phys., 2009, 81, 739.

38 C. Blanc, D. Fedorenko, M. Gross, M. In, M. Abkarian, M. A. Gharbi, J. B. Fournier, P. Galatola and M. Nobili, Phys. Rev. Lett., 2013, 111, 058302.

39 D. M. Kaz, R. McGorty, M. Mani, M. P. Brenner and V. N. Manoharan, Nat. Mater., 2011, 11, 138. 